\title{
Tuberculosis risk factors among tuberculosis patients in Kampala, Uganda: implications for tuberculosis control
}

Bruce J Kirenga ${ }^{1 *}$, Willy Ssengooba ${ }^{2,6}$, Catherine Muwonge ${ }^{3}$, Lydia Nakiyingi', Stephen Kyaligonza ${ }^{3}$, Samuel Kasozi ${ }^{3}$, Frank Mugabe ${ }^{3}$, Martin Boeree ${ }^{4}$, Moses Joloba ${ }^{2}$ and Alphonse Okwera ${ }^{5}$

\begin{abstract}
Background: Slow decline in the incidence of tuberculosis (TB) has been observed in most high TB burden countries. Knowledge of the prevalence of different TB risk factors can help expand TB control strategies. However with the exception of Human Immunodeficiency Virus (HIV) the prevalence of the other TB risk factors are poorly studied in Uganda. We aimed to determine the prevalence of different TB risk factors and TB disease presentation among TB patients in Kampala Uganda.
\end{abstract}

Methods: We assessed 365 adult TB patients and used descriptive statistics to summarize their socio-demographic, clinical, radiological, sputum mycobacteriology and TB risk factors (HIV, diabetes, TB contact, alcohol use, tobacco smoking, poverty and overcrowding) data.

Results: A total of 158 (43.3\%) patients were male and the median age was 29 (IQR 28-30). Majority of the patients (89.2\%) had pulmonary TB, 86.9\% were new and 13.2\% were retreatment. Wasting (i.e. body mass index of $<18.5 \mathrm{~kg} / \mathrm{m}^{2}$ ) was found in $38.5 \%$ of the patients and $63 \%$ presented with cough. Constitutional symptoms (fever, anorexia, night sweats and weight loss) were reported by $32.1 \%$. Most patients (78.6\%) presented with non-cavity lung parenchyma disease (infiltrates, nodules, masses) but 35.2\% had cavity disease. Pleural disease was detected in 19.3\% of patients. Positive smear microscopy and culture (irrespective of month of treatment) was found in 52.7\% and $36.5 \%$ of patients respectively. Any drug resistance was detected in $21.1 \%$ of patients while multidrug resistance (MDR) TB defined as resistance to rifampicin and isoniazid was detected in $6.3 \%$ of patients. All MDR patients were new patients. The prevalence of TB risk factors were as follows: HIV $41.4 \%$, diabetes $5.4 \%$, close contact $11.5 \%$, family history $17.5 \%$, smoking $26.37 \%$, poverty $39.5 \%$, overcrowding $57.3 \%$ and alcohol use $50.7 \%$. Overcrowding increased smear positive rate, prevalence ratio $1.22, p=0.09$ but all the other studied risk factors did not affect clinical, radiological and mycobacteriological study patient characteristics.

Conclusions: Among TB patients in Kampala, Uganda, there is high prevalence of the known TB risk factors. Targeting reducing their prevalence may lead to better TB control in the country. Tuberculosis, risk factors, Uganda.

\section{Background}

Uganda is one of the 22 high tuberculosis (TB) burden countries (HBC) in the world [1]. From an estimated population of 35 million people with national HIV prevalence of $7.3 \%, 45,546$ TB patients were diagnosed in the in the year 2010 of which $54 \%$ were

\footnotetext{
* Correspondence: brucekirenga@yahoo.co.uk

${ }^{1}$ Department of Medicine, Makerere University College of Health Sciences, P.O. Box 7072, Kampala, Uganda

Full list of author information is available at the end of the article
}

HIV-infected [1-4]. Of these 56\% were smear positive, $28 \%$ were smear negative and $11 \%$ had extra pulmonary TB [1].

Despite implementation of the $\mathrm{WHO}$ recommended directly observed therapy short course(DOTS)TB control strategy, the reductions in the incidence of TB have been minimal in $\mathrm{HBC}$ [5]. Because of this slow decline of TB incidence there is currently renewed interest in finding new TB control strategies. Focus has been on such strategies as adding to the current arsenal of TB drugs, finding a TB vaccine and designing shorter $\mathrm{TB}$

\section{Biomed Central}


regimens. However, knowledge of what makes some persons develop TB and others not (risk factors) has potential of helping further to refocus the search for novel public health TB control strategies. Reported TB risk factors include HIV infection, male sex, co-morbidities such as diabetes, and family history of $\mathrm{TB}$, absence of a Bacillus Calmette-Guérin (BCG) scar, smoking, alcohol use, single marital status, overcrowding, and poor socioeconomic status [6-9].

Infection with HIV has been the mostly associated risk factor for TB diseases in most HBC. [1] However, epidemiologic studies have found that not all TB cases can be attributed to HIV [10-13]. For example in Tanzania the population attributable fraction of TB due to HIV was $29 \%$ while in Nigeria it was reported as $40 \%[11,12]$. These findings highlight the importance of other TB risk factors in perpetuating TB epidemics. Two of the risk factors that are increasingly being recognized as major drivers of the TB epidemic are tobacco smoking and diabetes [14-16]. Basu et al. through a mathematical model have shown that if current smoking trends continue, tobacco smoking will be responsible for 18 million TB cases and 40 million deaths in the next 40 years [17]. A recent meta-analysis has found a strong association (relative risk of 3.1) between TB and diabetes [18]. The association between $\mathrm{TB}$, tobacco smoking and diabetes highlight the likely convergence of the emerging non communicable diseases (NCD) epidemic in developing countries and communicable diseases such as tuberculosis [19].

We conducted this study to determine the prevalence of known TB risk factors among TB patients diagnosed in Kampala district in Uganda and explore their effect on clinical, radiological and mycobacteriological TB disease presentation.

\section{Methods \\ Design and setting}

This was across sectional study of patients from selected government TB diagnostic and treatment units (DTUs) in Kampala district. Participating DTUs were: Kisenyi Health Center, Kiruddu Health Center, Kawaala Health Center, Kitebi Health Center, Kawempe Health Center, Kiswa Health Center and Mulago hospital. Kampala district was chosen because of its accessibility and large number of TB patients from all over the country $(25 \%$ of total national TB notifications are from Kampala district) [20].

\section{Study participants}

Participants were adult TB patients aged 18 years and above consecutively enrolled from participating DTUs during the period September 2012 through February 2013.

\section{Sample size calculation}

Because the prevalence of most of the studied risk factors was not known we assumed a $50 \%$ prevalence rate. Targeting a precision of 0.05 and standard normal deviation at 95\% confidence of 1.96 and allowing 10\% nonresponse rate we estimated that 423 patients would be required to obtain precise estimates of the prevalence of the studied risk factors. This was not attained due to slow enrolment and an unexpected TB drugs stock out during the study period that affected enrolment of patients who were on continuation phase of TB treatment.

\section{Study procedures}

Patients were interviewed using a structured questionnaire to collect demographic, socioeconomic and TB symptom data. All patients provided one sputum sample for mycobacteriological tests. At the study laboratory, smear microscopy was done by concentrated fluorescent microscopy method, after culture inoculation [21]. All samples were decontaminated for culture using standard operating procedures and all cultures were incubated for up to 8 weeks on two Lowenstein Jensen (LJ) media [22]. Culture results were communicated to the health facilities for patient management. A patient's culture result was considered contaminated only if all LJ slants had non-mycobacterial growth, otherwise, positive result was taken as a single colony on solid media and AFB on Ziehl-Neelsen microscopy and/or capilia TB $\mathrm{Neo}^{\mathrm{ma}}$ positive, if doubtful colonies were observed. The absence of colonies on LJ medium after 8 weeks was reported as a negative result. Positive cultures underwent Drug Susceptibility Testing (DST) testing to determine if drug resistance was present. DST was performed by Automated Mycobacterial Growth Indicator tube method (MGIT960) as previously described [22].

Only patients at TB treatment initiation (month 0 ) underwent chest X-ray (CXR) examination and fasting blood sugar testing. Blood sugar was measured by Cobas intergra 400 version 3.4.4.0809 machine. All patients were HIV tested according to the ministry of health rapid HIV testing algorithm [23].

\section{Definition of variables}

Tobacco smoking was defined as current or former use of at least 100 cigarettes in a life time [24]. Alcohol use was defined as history of drinking alcohol at least three times a week as previously used in other alcohol use studies in Uganda [24]. We defined poverty as a daily income of less than $1.25 \mathrm{USD} /$ day [25] while overcrowding was defined as two or more adult persons who are not of opposite sex sharing a room [26].

Diagnosis of diabetes was made according to American Diabetes Association cut off ranges $(<99 \mathrm{mg} / \mathrm{dL}$, normal, $100-125 \mathrm{mg} / \mathrm{dL}$, pre diabetes and $\geq 126 \mathrm{mg} / \mathrm{dL}$, diabetes) 
[27]. We did not consider symptoms to make a diagnosis of diabetes. Abnormal CXR was defined as any abnormality on the CXR. Chronic cough was defined as cough lasting two or more weeks.

\section{Data analysis}

Data were entered into an access database, cleaned and analyzed using epi-info software version 3.54. Descriptive statistics was used to summarize patients' socio-

\section{Table 1 Study patients' characteristics}

\begin{tabular}{|c|c|c|}
\hline Characteristic & Level & No $(\%)$ \\
\hline \multicolumn{3}{|c|}{ Demographic characteristics } \\
\hline \multirow[t]{3}{*}{ Age } & $<40$ & $296(81.1 \%)$ \\
\hline & $41-65$ & $68(18.6 \%)$ \\
\hline & $>65$ & $1(0.3 \%)$ \\
\hline \multirow[t]{2}{*}{ Gender } & Male & $158(43.3)$ \\
\hline & Female & $207(56.7)$ \\
\hline \multirow[t]{2}{*}{ Occupation } & Un/ self-employed & $110(64.0 \%)$ \\
\hline & Employed & $62(36.0 \%)$ \\
\hline \multirow[t]{4}{*}{ Education } & None & $19(5.2)$ \\
\hline & Primary & $110(30.1)$ \\
\hline & Secondary & $39(10.7)$ \\
\hline & Tertiary & $108(29.6)$ \\
\hline \multicolumn{3}{|l|}{ Clinical characteristics } \\
\hline \multirow[t]{2}{*}{ Site of TB (260) } & Pulmonary & $232(89.2)$ \\
\hline & Extrapulmonary & $28(10.8)$ \\
\hline \multirow[t]{2}{*}{ Treatment category } & New & $317(86.9)$ \\
\hline & Retreatment & $48(13.2)$ \\
\hline $\begin{array}{l}\mathrm{BMI}<18.5 \mathrm{~kg} / \mathrm{m} 2 \\
(\mathrm{n}=234)\end{array}$ & $<18.5$ & $90(38.5)$ \\
\hline \multirow[t]{2}{*}{ TB symptoms $(n=365)$} & Cough & $230(63.0)$ \\
\hline & Constitutional symptoms & $117(32.1)$ \\
\hline HIV infection $(n=365)$ & HIV infected & 153(41.9) \\
\hline \multicolumn{3}{|c|}{ Radiological characteristics } \\
\hline \multirow[t]{4}{*}{ CXR findings $(n=145)$} & $\begin{array}{l}\text { Parenchymal (nodules, } \\
\text { infiltrates, masses) }\end{array}$ & $114(78.6)$ \\
\hline & Cavities & $51(35.2)$ \\
\hline & $\begin{array}{l}\text { Pleural (pleural effusion, } \\
\text { pleural thickening) }\end{array}$ & $28(19.3)$ \\
\hline & Adenopathy (hilar \& mediastinal) & $4(2.8)$ \\
\hline \multicolumn{3}{|c|}{ Mycobacteriology characteristics } \\
\hline \multirow[t]{2}{*}{ Smear } & Negative & $123(47.3 \%)$ \\
\hline & Positive & 137 (52.7\%) \\
\hline \multirow{4}{*}{$\begin{array}{l}\text { Culture and drug } \\
\text { sensitivity }\end{array}$} & Negative & 165 (63.5\%) \\
\hline & Positive & 95 (36.5\%) \\
\hline & $\begin{array}{l}\text { Any drug resistance } \\
(\mathrm{INH}, \mathrm{R}, \mathrm{Z}, \mathrm{E})\end{array}$ & $20(21.1 \%)$ \\
\hline & MDR & $6(6.3 \%)$ \\
\hline
\end{tabular}

demographic, clinical, radiological and mycobacteriological characteristics. Prevalence of different risk factors was calculated as a proportion of those with the risk factor divided by the total number of patients who had data for that risk factor. Proportions of key clinical (chronic cough), mycobacteriology (smear positivity) and radiological (abnormal CXR) were calculated and compared between the different risk factors studied using a $t$-test. A $\mathrm{p}$ value of $<0.05$ was considered to represent significant difference.

\section{Ethics consideration}

All patients provided written informed consent. Ethical approval was obtained from the Makerere University School of Biomedical Science Research and Ethics committee and from the Uganda National Council of Science and Technology. Each patient was assigned a unique study number for identification. Patient's names were not used on any study related documents. Study documents were kept under lock and key.

\section{Results}

\section{Socio-demographic characteristics}

A total of 365 TB patients were recruited during the study period. One hundred and forty one $(38.6 \%)$ were at month zero, 84 (23.0\%) were at month two, 64 $(17.5 \%)$ were at month $5,33(9.0 \%)$ were at month six and $43(11.8 \%)$ were at month eight of TB treatment.

Study patients characteristics are shown in Table 1 . Male patients were 158 (43.3\%), median age was 29 (IQR 28-30) and 296 (81.1\%) were under the age 40 years.

Majority of the patients (89.2\%) had pulmonary TB, $86.9 \%$ were new and $13.2 \%$ were retreatment. $38.5 \%$ were wasted (i.e. body mass index of $<18.5 \mathrm{~kg} / \mathrm{m}^{2}$ ) and $63 \%$ presented with cough. Constitutional symptoms (fever, anorexia, night sweats and weight loss) were reported by $32.1 \%$.

Most (78.6\%) presented with either infiltrates, nodules or masses, $35.2 \%$ cavities and $19.3 \%$ of the patients had TB disease presenting with pleural effusion and/or thickening.

Positive smear microscopy and culture (irrespective of month of treatment) was found in $52.7 \%$ and $36.5 \%$ of patients respectively. Of the 95 patients with 95 isolates any drug resistance was detected in $21.1 \%$ of patients while multidrug resistance (MDR) TB defined as resistance to rifampicin and isoniazid was detected in $6.3 \%$ of patients. All MDR patients were new.

\section{TB risk factors}

The prevalence of each of the investigated TB risk factors is shown in Table 2. The prevalence of HIV infection was $41.4 \%$ (151 of 365 ) while that of diabetes was 


\begin{tabular}{lll}
$\begin{array}{l}\text { Table } 2 \text { Prevalence of studied tuberculosis risk factors } \\
\text { among study participants }\end{array}$ & $\begin{array}{l}\text { Number with } \\
\text { risk factor }\end{array}$ & \% (95\% Cl) \\
\hline Risk factor & 144 & $39.5(34.4-44.7)$ \\
\hline $\begin{array}{l}\text { Income below poverty line } \\
\text { (1.25 USD/day) }\end{array}$ & 209 & $57.3(52.0-62.4)$ \\
Overcrowding (>2 persons/room) & $26.3(21.9-31.1)$ \\
Former smoker & 96 & $1.4(0.4-3.2)$ \\
Current smoker & 5 & $50.7(45.4-55.9)$ \\
Alcohol use & 185 & $41.4(36.3-46.6)$ \\
HIV infection & 151 & $11.5(8.4-15.2)$ \\
TB contact & 42 & $17.5(13.8-21.8)$ \\
Family history of TB & 64 & $5.4(3.1-6.7)$ \\
Diabetes & 7 & \\
\hline
\end{tabular}

5.4\% (7 of 130). Although only 7 patients had blood sugars levels diagnostic of diabetes, another 12 patients (9.2\%) had blood sugar levels diagnostic of pre-diabetes.

Current smoking was 1.4\% (5 of 365) but history of smoking was reported by at $26.3 \%$ (96 of 365) of the patients and $52.6 \%$ of former smokers reported to have quit in the past one year.

Poverty was found in $39.5 \%$ (144 of 365) while overcrowding was reported by $57.3 \%$ (209 of 365) patients. One hundred and eighty five patients $(50.7 \%)$ reported alcohol use.

More patients (57.6\%) from overcrowded homes were smear positive compared to those from homes without overcrowding (47.1\%), prevalence ratio (PR) of 1.22 , $\mathrm{p}=0.09$. We however found no other significant differences in the clinical, radiological and mycobacteriological TB disease presentation between other risk factors (Table 3).

\section{Discussion}

In this cross sectional study among TB patients under care in Kampala, Uganda we have established the prevalence of key known TB risk factors as follows: HIV infection $41.4 \%$, alcohol use $50.7 \%$, poverty $39.5 \%$, smoking $26.3 \%$, family history of TB $17.5 \%$, TB contact $11.5 \%$ and diabetes $5.4 \%$.

High prevalence of HIV among TB patients is due to HIV associated immune suppression which increases risk for $\mathrm{TB}$ through reactivation of latent $\mathrm{TB}$ disease and increased proportion of persons who progress from $\mathrm{TB}$ infection to disease. The annual risk of TB among HIV patients is $10 \%$ /year compared to the $10 \%$ life time risk among HIV negative persons [28]. The prevalence of HIV found in this study is about 6 times (41.4 vs.7.3) that in the general population. The prevalence is however lower than the over $50 \%$ prevalence previously reported [1]. This difference could be because of the small sample size and small number of recruitment sites. However, a study investigating prevalence of MDR-TB by Lukoye D et al. reported lower HIV prevalence of $30.5 \%$ compared to the current study [29].

The association between tobacco smoking and TB is well described $[15,17,30]$. The prevalence we report in this study does not differ significantly from those reported in other studies in Africa [31] but differs from those in other regions [32]. For example a study in South Africa reported a prevalence of $21.8 \%$ while a study in Malaysia reported a prevalence of $40.3 \%$ [33]. These differences could be attributed to background smoking prevalence in the general population. The prevalence of background tobacco smoking in Uganda population is limited because population based surveys have not been conducted. A review conducted by Nturibi EM et al. identified three studies on tobacco smoking prevalence in Uganda [34]. The prevalence in these studies ranged from $5.3 \%$ to $21.9 \%$ [35]. It must be noted all the three studies included mainly young people in schools. An unpublished fact sheet from the recently concluded GATS tobacco use survey in Uganda reported a prevalence of $12 \%$ in the general population (http://www.afro.who. int/en/uganda/press-materials/item/6713-uganda-releasesresults-of-the-global-adult-tobacco-survey-2013.html). We also noted that the prevalence of current smoking was only $1.37 \%$ but on further evaluation it was found that about half of the former smokers had actually quit in the past year. This could be due to fact that most patients stopped smoking when they developed TB respiratory symptoms interpreting them as due to smoking.

Previous studies have documented a strong association of diabetes and TB $[18,36]$. A recent meta-analysis has found a strong association (relative risk of 3.1) between TB and diabetes [18]. The prevalence reported in this study is lower than that reported in a recent study among hospitalized TB patients in Mulago Hospital, Kampala [34]. This difference could be due to the fact that stress hyperglycemia in the hospitalized patients may have contributed to more patients being diagnosed with diabetes. The study also used random blood sugar instead of the recommended fasting blood sugar. In addition 12 patients met the criteria of pre-diabetes. Taking the pre-diabetes and the diabetes together gives a prevalence of $10.8 \%$ comparable to what was found in the Mulago hospital study.

We investigated the number of patients whose daily incomes were below the poverty line and how many were living in overcrowded homes. The prevalence of poverty we found $(39.5 \%)$ was slightly higher than the national average of 38\% [37]. We note that this study was conducted in the capital city of Uganda. Therefore patients' incomes may be higher than 1.25 USD/day designated by the World Bank. In the 2012-2013 when the study was conducted $1.25 \mathrm{USD} /$ day could barely afford a 
Table 3 Tuberculosis risk factors and selected tuberculosis disease presentation

\begin{tabular}{|c|c|c|c|c|c|c|}
\hline $\begin{array}{l}\text { Independent } \\
\text { variables }\end{array}$ & $\begin{array}{l}\text { Smear positive } \\
\mathrm{N}(\%)\end{array}$ & $\begin{array}{l}\text { PR (SE), } \\
p \text { value }\end{array}$ & $\begin{array}{l}\text { Abnormal chest x-ray } \\
\text { N (\%) }\end{array}$ & $\begin{array}{l}\text { PR (SE), } \\
p \text { value }\end{array}$ & $\begin{array}{l}\text { Chronic cough } \\
N(\%)\end{array}$ & $\begin{array}{l}\text { PR (SE), } \\
p \text { value }\end{array}$ \\
\hline \multicolumn{7}{|l|}{ Poverty } \\
\hline Yes & $17(53.1)$ & $1.10(0.21)$ & $12(37.5)$ & $1.07(0.28)$ & $15(46.9)$ & $0.81(0.16$ \\
\hline No & $61(48.4)$ & 0.625 & $44(34.9)$ & 0.783 & 73 (57.9) & 0.296 \\
\hline \multicolumn{7}{|l|}{ Overcrowding } \\
\hline Yes & $80(57.6)$ & $1.22(0.15)$ & $54(38.9)$ & $1.04(0.17)$ & $80(57.6)$ & $1.09(0.12)$ \\
\hline No & $57(47.1)$ & 0.097 & $45(37.2)$ & 0.784 & $64(52.9)$ & 0.453 \\
\hline \multicolumn{7}{|l|}{ Smoking } \\
\hline Yes & $45(58.4)$ & $1.16(0.14)$ & $27(35.1)$ & $0.89(0.16)$ & $40(52.0)$ & $0.92(0.12)$ \\
\hline No & $92(50.6)$ & 0.230 & 72 (39.6) & 0.503 & $103(56.6)$ & 0.501 \\
\hline \multicolumn{7}{|l|}{ Alcohol use } \\
\hline Yes & 77 (55.8) & $1.13(0.14)$ & $50(36.2)$ & $0.90(0.14)$ & $78(56.5)$ & $1.04(0.12)$ \\
\hline No & $60(49.2)$ & 0.290 & $49(40.2)$ & 0.514 & $66(54.1)$ & 0.695 \\
\hline \multicolumn{7}{|l|}{ HIV infection } \\
\hline Positive & $128(52.9)$ & $1.1(0.26)$ & $90(37.2)$ & $0.74(0.19)$ & $137(56.6)$ & $1.46(0.44)$ \\
\hline Negative & $9(50.0)$ & 0.817 & $9(50.0)$ & 0.237 & $7(38.9)$ & 0.212 \\
\hline
\end{tabular}

$\mathrm{PR}=$ Prevalence Ratio, $\mathrm{SE}=$ Standard Error.

simple lunch meal. In essence this cut off may grossly underestimate the poverty levels of the populace especially those in urban areas. Uganda with $53 \%$ of the population living in overcrowded homes is number 14 on the list of the 20 countries with most overcrowded homes in the world [26]. Overcrowding has been previously documented as a strong risk for TB [38]. In this study we found that $57.3 \%$ of the study participants were living in overcrowded homes. This is higher than the national average. The national figures do not take into account the TB burden in the community. The study area (Kampala) has the highest prevalence of TB in the country. One in four TB cases notified in Uganda are from Kampala [20]. Therefore overcrowding may represent a greater risk of $\mathrm{TB}$ than in areas where the $\mathrm{TB}$ is much lower.

Close contact is another well described risk factor for TB [39-44]. Close contact was estimated to account for 9-13\% of the TB cases in Malawi [44]. In a systematic review by Fox GJ et al. the prevalence of active TB and latent $\mathrm{TB}$ infection among $\mathrm{TB}$ contacts was $3.1 \%$ and $51.5 \%$ respectively [40]. A prevalence of 3.1 represents a major increase in TB prevalence compared to the general population. For example in Uganda TB prevalence is $175 / 100,000$ population $(0.02 \%)$ [1]. It follows therefore that $\mathrm{TB}$ prevalence among contacts is very high [1]. The odds ratio of having a family member with $\mathrm{TB}$ and developing $\mathrm{TB}$ is estimated at 13.4 further highlighting the importance of close TB contact and TB risk [6]. This factor is not well studied among patients in most TB programs. We contend that a prevalence of family history of TB of 17.5 is high and need further investigations. This would mean that beyond close contacts health workers would elicit family history of TB, the presence of which would refine targeting of TB screening strategies.

We also investigated the prevalence of alcohol use among TB patients. The association of TB and alcohol is well described in the literature $[45,46]$. It can be hypothesized that it is due to the close contact that takes place in bars as opposed to other social gatherings. Lanoroth $\mathrm{K}$ et al. conducted a systematic review in which they established that alcohol use was independently associated with TB (RR 2.94). More than $50 \%$ of the TB patients in our study consumed alcohol. Although we investigated the effect of the studied risk factors on TB disease presentation we did not find significant influence of the risk factors on disease presentation. Marginal influence was observed between overcrowding and smear positive rate. This could be due to the fact that our study was not powered to detect these differences. Secondly we enrolled patients at different stages of treatment which could have affected the prevalence of the outcomes variables.

Lastly we found an MDR rate of $6.3 \%$ and all were from new patients. This prevalence is much higher than that found in the national drug resistance survey conducted in 2010 (1.4\%) and that found in the Kampala drug resistance survey conducted in 2008 (1.1\%) [29,47]. This difference could be due to smaller sample size since we had only 95 culture positive patients. It could also be sampling bias because we included only government 
DTUs and enrolled patients during one reporting quarter. We also included TB patients irrespective of duration on TB treatment. Therefore some new patients could have started developing drug resistance during the time they were on TB treatment. We also noted that during the study period there were drug stock outs at study clinics. This could have further facilitated emergence of drug resistance among study patients. These shortcomings not withstanding these results emphasize the role of surveillance of drug resistance. A follow up survey or analysis of routinely collected data can help clarify if there could be increase in drug resistance in the country.

This study had some limitations. Firstly the patients were recruited over one quarter of the reporting year. It is possible that there may be differences in the patients who reported to the health facilities during other times. We defined overcrowding using alternative method of number of persons per room. This method does not take into account the size of the room. This was done because we could not obtain accurate room sizes in order to calculate the living area per person. Patient incomes were also self -reported. We used daily income as a measure of poverty. However we recognize that a more extensive investigation of poverty parameters would provide different estimates. We did not take into consideration symptoms while making a diagnosis of diabetes as recommended. Only $86 \%$ of the estimated sample size was achieved under convenient sampling, future studies with bigger sample size are recommended. Nevertheless, much as this study is more qualitative than quantitative, it is one of few studies specifically conducted to investigate the prevalence of TB risk factors among TB patients. It has generated data that should guide more detailed, possibly better designed quantitative studies.

\section{Conclusions}

Among TB patients in Kampala, Uganda, there is high prevalence of the known TB risk factors. Targeting reducing their prevalence may lead to better TB control in the country.

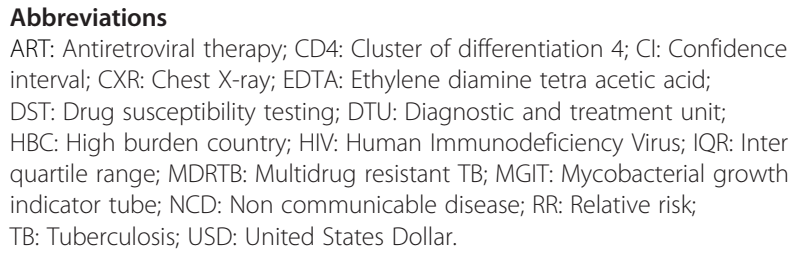

Abbreviations

ART: Antiretroviral therapy; CD4: Cluster of differentiation 4; Cl: Confidence interval; CXR: Chest X-ray; EDTA: Ethylene diamine tetra acetic acid; DST: Drug susceptibility testing; DTU: Diagnostic and treatment unit; HBC: High burden country; HIV: Human Immunodeficiency Virus; IQR: Inter quartile range; MDRTB: Multidrug resistant TB; MGIT: Mycobacterial growth indicator tube; NCD: Non communicable disease; RR: Relative risk: TB: Tuberculosis; USD: United States Dollar.

\section{Competing interests}

The authors declare that they have no competing interests. I, Dr. Bruce J Kirenga, the corresponding author, had full access to all of the data in the study and I take responsibility for the integrity of the data and the accuracy of the data analysis.

\section{Authors' contributions}

AO, MJ, BJK, WS, LN and CM conceived and wrote the study protocol. AO and MJ obtained funding for the study. All authors participated in study data acquisition, analysis and manuscript preparation. All authors read and approved the final manuscript.

\section{Acknowledgements}

The authors would like to acknowledge the invaluable contribution made by the research assistants and health workers at the recruitment sites. Special thanks go to Geraldine Nalwadda for providing excellent administrative support to the project.

\section{Author details}

${ }^{1}$ Department of Medicine, Makerere University College of Health Sciences, P.O. Box 7072, Kampala, Uganda. ${ }^{2}$ Department of Microbiology, Makerere University College of Health Sciences, Kampala, Uganda. ${ }^{3}$ National Tuberculosis and Leprosy Program, Ministry of Health, Kampala, Uganda. ${ }^{4}$ Department of Pulmonary Diseases, Radboud University Nijmegen Medical Center, Nijmegen, The Netherlands. ${ }^{5}$ National Tuberculosis treatment unit, Mulago Hospital, Kampala, Uganda. ${ }^{6}$ Department of Global Health and Amsterdam Institute of Global Health and Development, Academic Medical Center, University of Amsterdam, P.O. Box 22700, 1100 DE Amsterdam, Netherlands.

Received: 14 March 2014 Accepted: 8 January 2015

Published online: 21 January 2015

\section{References}

1. Global TB control report 2012. Available at [http:/apps.who.int/iris/bitstream/ 10665/75938/1/9789241564502_eng.pdf]. Accessed 12 December 17, 2014.

2. Uganda; City population. Available at http://www.citypopulation.de/Uganda. html. Accessed on 12 December 17, 2014.

3. UNdata | country profile | Uganda. Available at [http://data.un.org/ CountryProfile.aspx?crName = Uganda]. Accessed on 12 December 17, 2014

4. Uganda AIDS Indicator Survey 2011 - Key findings Available at [http:// health.go.ug/docs/UAIS_2011_KEY_FINDINGS.pdf]. Accessed on 12 December 17, 2014.

5. THE GLOBAL PLAN TO STOP TB 2011-2015. Available at [http://stoptb.org/ assets/documents/global/plan/TB_GlobalPlanToStopTB2011-2015.pdf]. Accessed on 12 December 17, 2014.

6. Lienhardt C, Fielding K, Sillah J, Bah B, Gustafson P, Warndorff D, et al. Investigation of the risk factors for tuberculosis: a case-control study in three countries in West Africa. Int J Epidemiol. 2005;34(4):914-23.

7. Lienhardt C, Fielding K, Sillah J, Tunkara A, Donkor S, Manneh K, et al. Risk factors for tuberculosis infection in Sub-Saharan Africa. Am J Respir Crit Care Med. 2003;168(4):448-55.

8. Davies P. Risk factors for tuberculosis. Monaldi Arch Chest Dis. 2005;63 (1):37-46.

9. Coker R, McKee M, Atun R, Dimitrova B, Dodonova E, Kuznetsov S, et al. Risk factors for pulmonary tuberculosis in Russia: case-control study. BMJ. 2006;332(7533):85-7

10. van Cleeff MR, Chum HJ. The proportion of tuberculosis cases in Tanzania attributable to human immunodeficiency virus. Int J Epidemiol. 1995;24 (3):637-42.

11. Van den Broek J, Borgdorff MW, Pakker NG, Chum HJ, Klokke AH, Senkoro $\mathrm{KP}$, et al. HIV-1 infection as a risk factor for the development of tuberculosis: a case-control study in Tanzania. Int J Epidemiol. 1993;22(6):1159-65.

12. Richards SB, St Louis ME, Nieburg P, Coulibaly IM, Coulibaly D, Abouya L, et al. Impact of the HIV epidemic on trends in tuberculosis in Abidjan, Cote d'Ivoire. Tuber Lung Dis. 1995;76(1):11-6.

13. Corbett EL, Bandason T, Cheung YB, Munyati S, Godfrey-Faussett P, Hayes R, et al. Epidemiology of tuberculosis in a high HIV prevalence population provided with enhanced diagnosis of symptomatic disease. PLoS Med 2007:4(1).

14. Bates MN, Khalakdina A, Pai M, Chang L, Lessa F, Smith KR. Risk of tuberculosis from exposure to tobacco smoke: a systematic review and meta-analysis. Arch Intern Med. 2007;167(4):335-42.

15. Gambhir HS, Kaushik RM, Kaushik R, Sindhwani G. Tobacco smoking-associated risk for tuberculosis: a case-control study. Int Health. 2010;2(3):216-22.

16. Dooley KE, Chaisson RE. Tuberculosis and diabetes mellitus: convergence of two epidemics. Lancet Infect Dis. 2009;9(12):737-46. 
17. Basu S, Stuckler D, Bitton A, Glantz SA. Projected effects of tobacco smoking on worldwide tuberculosis control: mathematical modelling analysis. Bmj 2011;4(343).

18. Jeon CY, Murray MB. Diabetes mellitus increases the risk of active tuberculosis: a systematic review of 13 observational studies. PLoS Med. 2008;5(7):0050152.

19. Creswell J, Raviglione M, Ottmani S, Migliori GB, Uplekar M, Blanc L, et al. Tuberculosis and noncommunicable diseases: neglected links and missed opportunities. Eur Respir J. 2011;37(5):1269-82.

20. NTLP. Cohort data, Uganda National TB and Leprosy Program. 2010.

21. CDC. Public health mycobacteriology: a guide for the level III laboratory control CFD. In: Department of Health EAW. Atlanta, Georgia: Public Health Service; 1985.

22. Siddiqi SH. MGIT Procedure Manual; For BACTEC MGIT 960 TB System, Specially Prepared for FIND MGIT demonstration Project. 2006. Available; http://www.finddiagnostics.org/export/sites/default/resource-centre/ find_reports/pdfs/mgit_manual_nov_2007.pdf.

23. Uganda Ministry of Health. Uganda National policy guidelines for HIV counseling and testing. Kampala, Uganda: Ugandan Ministry of Health; 2003. http://www.who.int/hiv/pub/guidelines/uganda_art.pdf lines/ uganda_art.pdf Accessed November 2012.

24. Tumwesigye NM, Kasirye R. Gender and the major consequences of alcohol consumption in Uganda. In: Obot I, Robin R, editors. Alcohol, gender and drinking problems. World Health Organization: Geneva; 2006. p. 189-208.

25. Ravallion M, Chen S, Sangraula P. Dollar a day revisited. World Bank Econ Rev. 2009;23(2):163-84.

26. Overcrowding. World Health Organisation. Available at [http://cdrwww.who, int/ceh/indicators/overcrowding.pdf]. Accessed on 12 December 17, 2014

27. Diagnosis and classification of diabetes mellitus. Diabetes Care 2010, 33(1):dc10-S062.

28. Cole EC, Cook CE. Characterization of infectious aerosols in health care facilities: an aid to effective engineering controls and preventive strategies. Am J Infect Control. 1998;26(4):453-64.

29. Lukoye D, Adatu F, Musisi K, Kasule GW, Were W, Odeke R, et al. Anti-tuberculosis drug resistance among new and previously treated sputum smear-positive tuberculosis patients in Uganda: results of the first national survey. PLoS One. 2013;8(8):e70763.

30. Ryan $\mathrm{H}$, Trosclair A, Gfroerer J. Adult current smoking: differences in definitions and prevalence estimates_NHIS and NSDUH, 2008. J Environ Public Health 2012;91:83-68.

31. Louwagie GM, Ayo-Yusuf OA. Tobacco use patterns in tuberculosis patients with high rates of human immunodeficiency virus co-infection in South Africa. BMC Public Health. 2013;13(1031):1471-2458.

32. Wang J, Shen H. Review of cigarette smoking and tuberculosis in China: intervention is needed for smoking cessation among tuberculosis patients. BMC Public Health. 2009;9(292):1471-2458.

33. Awaisu A, Nik Mohamed MH, Abd Aziz N, Syed Sulaiman SA, Mohamad Noordin N, Muttalif AR, et al. Tobacco use prevalence, knowledge, and attitudes among newly diagnosed tuberculosis patients in Penang State and Wilayah Persekutuan Kuala Lumpur, Malaysia. Tob Induc Dis. 2010;8 (1):1617-9625

34. Kibirige D, Ssekitoleko R, Mutebi E, Worodria W. Overt diabetes mellitus among newly diagnosed Ugandan tuberculosis patients: a cross sectional study. BMC Infect Dis. 2013;13(122):1471-2334.

35. Nturibi EM, Akinsola AK, McCurdy SA. Smoking prevalence and tobacco control measures in Kenya, Uganda, the Gambia and Liberia: a review. Int J Tuberc Lung Dis. 2009;13(2):165-70.

36. Young F, Wotton CJ, Critchley JA, Unwin NC, Goldacre MJ. Increased risk of tuberculosis disease in people with diabetes mellitus: record-linkage study in a UK population. J Epidemiol Community Health. 2012;66(6):519-23.

37. Poverty headcount ratio at $\$ 1.25$ a day (PPP) (\% of population). The world Bank Available at [http://data.worldbank.org/indicator/SI.POV.DDAY]. Accessed on 12 December 17, 2014.

38. Lienhardt C. From exposure to disease: the role of environmental factors in susceptibility to and development of tuberculosis. Epidemiol Rev. 2001;23 (2):288-301.

39. Cook VJ, Sun SJ, Tapia J, Muth SQ, Arguello DF, Lewis BL, et al. Transmission network analysis in tuberculosis contact investigations. J Infect Dis. 2007;196 (10):1517-27.

40. Webb RM, Holcombe M, Pearson MM. Tuberculosis contact investigation in a rural state. Int J Tuberc Lung Dis. 2003;7(12 Suppl 3):S353-7.
41. Fox GJ, Barry SE, Britton WJ, Marks GB. Contact investigation for tuberculosis: a systematic review and meta-analysis. Eur Respir J. 2013;41(1):140-56.

42. Xu C, Hu B. Prevalence of active pulmonary tuberculosis among household contacts of recently diagnosed pulmonary tuberculosis patients with positive sputum-smear. Zhonghua Liu Xing Bing Xue Za Zhi. 2008;29(7):693-5.

43. Singh J, Sankar MM, Kumar S, Gopinath K, Singh N, Mani K, et al. Incidence and prevalence of tuberculosis among household contacts of pulmonary tuberculosis patients in a peri-urban population of South Delhi, India. PloS one 2013, 8(7).

44. Crampin AC, Glynn JR, Traore H, Yates MD, Mwaungulu L, Mwenebabu M, et al. Tuberculosis transmission attributable to close contacts and HIV status. Malawi Emerg Infect Dis. 2006;12(5):729-35.

45. Rehm J, Samokhvalov AV, Neuman MG, Room R, Parry C, Lonnroth K, et al. The association between alcohol use, alcohol use disorders and tuberculosis (TB). A systematic review. BMC Public Health. 2009;9(450):1471-2458.

46. Nava-Aguilera E, Andersson N, Harris E, Mitchell S, Hamel C, Shea B, et al. Risk factors associated with recent transmission of tuberculosis: systematic review and meta-analysis. Int J Tuberc Lung Dis. 2009;13(1):17-26.

47. Deus L, Frank GJC, Nicholas E, Samuel K, Adatu FE, Lule JK, et al. Rates of anti-tuberculosis drug resistance in Kampala-Uganda are low and not associated with HIV infection. PLoS One. 2011;6(1):e16130.

\section{Submit your next manuscript to BioMed Central and take full advantage of:}

- Convenient online submission

- Thorough peer review

- No space constraints or color figure charges

- Immediate publication on acceptance

- Inclusion in PubMed, CAS, Scopus and Google Scholar

- Research which is freely available for redistribution 